\title{
Evaluation of Strontium-Containing PCL-PDIPF Scaffolds for Bone Tissue Engineering: In Vitro and In Vivo Studies
}

\author{
Agustina Berenice Lino, ${ }^{1}$ Antonio Desmond McCarthy, ${ }^{1}$ and Juan Manuel Fernández ${ }^{1,2}$ \\ ${ }^{1}$ LIOMM (Laboratorio de Investigación en Osteopatías y Metabolismo Mineral) - Departamento de Ciencias Biológicas, \\ Facultad de Ciencias Exactas, Universidad Nacional de La Plata, Calle 47 y 115 (1900), La Plata, Argentina; and ${ }^{2}$ Cátedra \\ Bioquímica Patológica, Facultad de Ciencias Exactas, Universidad Nacional de La Plata, Calle 47 y 115 (1900), La Plata, \\ Argentina
}

(Received 2 September 2018; accepted 4 December 2018; published online 17 December 2018)

Associate Editor Dr. Smadar Cohen oversaw the review of this article.

\begin{abstract}
Bone tissue engineering (BTE) has the general objective of restoring and improving damaged bone. A very interesting strategy for BTE is to combine an adequate polymeric scaffold with an osteoinductive compound. Strontium is a divalent cation that can substitute calcium in hydroxyapatite and induce both anabolic and anti-catabolic effects in bone. On the other hand, systemic increases in $\mathrm{Sr}^{2+}$ levels can provoke adverse cardiovascular effects. In the present study we have developed a compatibilized blend of poly- $\varepsilon$-caprolactone (PCL) and polydiisopropyl fumarate (PDIPF) enriched with $1 \%$ or $5 \% \mathrm{Sr}^{2+}$ and evaluated the applicability of these biomaterials for BTE, both in vitro and in vivo. In vitro, whereas Blend $+5 \% \mathrm{Sr}^{2+}$ was proinflammatory and anti-osteogenic, Blend $+1 \% \mathrm{Sr}^{2+}$ released very low quantities of the cation; was not cytotoxic for cultured macrophages; and showed improved osteocompatibility when used as a substratum for primary cultures of bone marrow stromal cells. In vivo, implants with Blend + $1 \% \mathrm{Sr}^{2+}$ significantly increased bone tissue regeneration and improved fibrous bridging (vs. Blend alone), while neither inducing a local inflammatory response nor increased serum levels of $\mathrm{Sr}^{2+}$. These results indicate that our compatibilized blend of PCL-PDIPF enriched with $1 \%$ $\mathrm{Sr}^{2+}$ could be useful for BTE.
\end{abstract}

Keywords-Bone marrow stromal cells, RAW 264.7 macrophages, Poly-e-caprolactone, Polydiisopropyl fumarate, Strontium, Bone regeneration.

Address correspondence to Juan Manuel Fernández, LIOMM (Laboratorio de Investigación en Osteopatías y Metabolismo Mineral) - Departamento de Ciencias Biológicas, Facultad de Ciencias Exactas, Universidad Nacional de La Plata, Calle 47 y 115 (1900), La Plata, Argentina. Electronic mail: jmfernandez@biol.unlp.edu.ar

\section{INTRODUCTION}

Most of the alternatives that currently exist to replace or restore extensively damaged bone tissue have several disadvantages. Metallic devices either lack or have low osseointegration; ceramics are fragile with low flexural strength; and bone grafts have low availability due to insufficient donors, show graft rejection and potentially can transmit diseases. In addition, the number of bone lesions is increasing due to greater life expectancy and population growth. ${ }^{4,22,28,31}$

Using concepts from different areas (engineering, medicine, materials science, biochemistry), Bone tissue engineering or BTE has the general objective of restoring and improving damaged bone. ${ }^{20}$ At present, several synthetic and natural polymers have been evaluated as scaffolds for use in BTE. ${ }^{8,19,21}$ Not all polymers can be used to design scaffolds; they must meet a number of requirements such as biocompatibility, low toxicity, adequate biodegradation rate and appropriate biomechanical properties. ${ }^{18}$

We have previously developed and characterized an ultrasound-compatibilized blend of two synthetic polymers, poly-e-caprolactone (PCL) and polydiisopropyl fumarate (PDPIF), that shows mechanical characteristics similar to trabecular bone. MC3T3-E1 preosteoblastic cells were able to deposit more apatite mineral on this blend than on the individual polymers, and in addition the scaffold showed no evidence of cytotoxicity. ${ }^{11,13}$

The ranelic salt of strontium has been used as a treatment for patients with osteoporosis and shows a dual effect on bone, both anabolic and anti-catabolic. ${ }^{23}$ Strontium ions can exert their bone-forming actions by activation of the calcium-sensing receptor, thus 
inducing genes such as Runx2 related to osteoblastic proliferation and maturation. ${ }^{15}$ Although strontium ranelate has recently been discontinued due to adverse systemic cardiovascular side-effects, there is evidence that strontium ions could be safely used to promote local bone repair when they are included in scaffolds designed for BTE. ${ }^{30}$ In these cases, it is important to verify that $\mathrm{Sr}^{2+}$ release from the scaffold is negligible, so as to preclude any systemic adverse effects.

In the present study, we have engineered strontiumcontaining PCL/PDIPF composite scaffolds, and analyzed their release of $\mathrm{Sr}^{2+}$, osteocompatibility and cytotoxicity properties, in order to evaluate their possible application for BTE. In addition, we have compared the efficacy of PCL/PDIFP scaffolds, with or without $\mathrm{Sr}^{2+}$, as scaffolds for bone tissue repair in animals with surgically-induced critical parietal lesions.

\section{MATERIALS AND METHODS}

\section{Preparation of Composite Scaffolds}

PCL was purchased from Aldrich and had a weightaverage molecular weight (MW) and polydispersity index (PI) of $65,000 \mathrm{~g} \mathrm{~mol}^{-1}$ and 1.4, respectively, as indicated by the manufacturer. PDIPF was synthesized by microwave-assisted radical polymerization, using benzoyl peroxide as the initiator, as we have previously described. $^{6}$ The MW and PI of PDIPF were $131,000 \mathrm{~g} \mathrm{~mol}^{-1}$ and 2.0, respectively, as determined by size exclusion chromatography (SEC). Compatibilized blends of PCL and PDIPF (75:25 wt\%; Blend) were obtained by ultrasound using a Bandelin HD60 equipment at $20^{\circ} \mathrm{C}$, following our previously described methodology. ${ }^{13}$ Strontium chloride was purchased from Sigma-Aldrich.

Blend films containing 0,1 and $5 \% \mathrm{Sr}^{2+}(\mathrm{w} / \mathrm{w})$ were obtained by dissolution in chloroform and casting in a glass Petri dish. The solvent was allowed to evaporate at room temperature and the resulting films were dried under vacuum until constant weight. For in vivo and in vitro experiments, films were cut to size and then sterilized by UV exposure for 2 h..$^{10,13}$

\section{Biocompatibility Studies with Bone Marrow Stromal Cells}

Bone marrow stromal cells (BMSC) were obtained from young male WKAH/Hok Wistar rats as we have described previously. They were characterized by their ability to differentiate into various phenotypes such as osteoblasts, adipocytes and chondrocytes (data not shown). ${ }^{27}$ Briefly, animals were sacrificed under anes- thesia by rapid neck dislocation. BMSC were collected by flushing the dissected femoral and tibial diaphysis medullary canal with DMEM (Invitrogen, Buenos Aires, Argentina) under sterile conditions. The resulting suspension was seeded in a $25 \mathrm{~cm}^{2}$ tissue culture flask. Cells were grown in DMEM supplemented with $5 \%(\mathrm{v} / \mathrm{v})$ fetal bovine serum (FBS, Natocor, Argentina) and antibiotics $(100 \mathrm{U} / \mathrm{mL}$ penicillin and $100 \mu \mathrm{g} /$ $\mathrm{mL}$ streptomycin) in a humidified atmosphere of $95 \%$ air and $5 \% \mathrm{CO}_{2}$. For the experiments, polymeric films were cut to size and placed in 24- or 48-well plates. BMSC were then re-suspended, plated on each film slice at a density of $5 \times 10^{4}$ cells/well and cultured in $10 \%$ FBS-DMEM at $37^{\circ} \mathrm{C}$. The viability of BMSC grown on scaffolds was estimated using a colorimetric tetrazolium assay. This assay measures the reduction of the tetrazolium salt 3-(4,5-dimethylthiazol-2-yl)-2,5diphenyl tetrazolium bromide (MTT) to formazan by intact mitochondria in living cells. Thus, absorbance change is directly proportional to the number of viable cells. ${ }^{16}$ For this assay, $3 \times 10^{4}$ BMSC were plated in a 48-well plate (with a polymer film placed in each well) and cultured for $24 \mathrm{~h}$ in $10 \%$ FBS-DMEM at $37^{\circ} \mathrm{C}$. The medium was removed, cells were washed with PBS and fresh medium containing the MTT reagent (Sigma, USA) at a final concentration of $1 \mathrm{mg} / \mathrm{mL}$ was added. After a 3-h incubation each film was removed, placed in a new 48-multiwell plate and washed again with PBS. Color was developed by addition of $200 \mu \mathrm{L}$ dimethylsulfoxide (DMSO) (Merck, Argentina) and mixing in a plate shaker for $20 \mathrm{~min}$, after which absorbance was measured at $540 \mathrm{~nm} .^{11}$

In other experiments, after cells reached confluence in 24-well plates (with a polymer film placed in each well) they were induced to differentiate into osteoblasts using an osteogenic medium (DMEM-10\% FBS containing $25 \mathrm{mg} / \mathrm{mL}$ ascorbic acid and $5 \mathrm{mM}$ sodium $\beta$ glycerol-phosphate), which was changed twice a week. Osteoblastic differentiation was evaluated by measuring alkaline phosphatase activity (ALP) and type 1 collagen production. After 14 days of osteogenic differentiation, for ALP determination cell monolayers were washed with phosphate-buffered saline (PBS) and a total cell extract was obtained by dissolution with $200 \mu \mathrm{L} 0.1 \%$ Triton-X100. A $100 \mu \mathrm{L}$ aliquot of the extract was used to evaluate ALP by hydrolysis of $\mathrm{p}$ nitrophenylphosphate into p-nitrophenol (p-NP) at $37^{\circ} \mathrm{C}$ for $1 \mathrm{~h}$. The absorbance of $\mathrm{p}$-NP was recorded at $405 \mathrm{~nm}^{7}$ Aliquots of each cell extract were used for protein determination by Bradford's technique. ${ }^{5}$ For evaluation of type 1 collagen production, after 14 days of osteogenic differentiation cells were fixed with Bouin's solution and stained with Sirius Red dye for $1 \mathrm{~h}$. The stained material was dissolved in $1 \mathrm{ml} 0.1 \mathrm{~N}$ 
sodium hydroxide and the absorbance of the solution was recorded at $550 \mathrm{~nm} .^{12}$

\section{Cytotoxicity-Induced NO Production by RAW 264.7 Macrophages}

Mouse macrophage RAW 264.7 cells were grown in DMEM supplemented with 5\% FBS and antibiotics (100 U/mL penicillin and $100 \mu \mathrm{g} / \mathrm{mL}$ streptomycin) in a humidified atmosphere of $95 \%$ air and $5 \% \mathrm{CO}_{2}$. For the experiments, polymeric films were cut to size, inserted in each well of 24-well plates, and macrophages were plated on the films. Nitric oxide (NO) production was assessed using Griess reagent. ${ }^{25}$ The stable endproduct of $\mathrm{NO}$ and nitrite released into the culture medium by RAW 264.7 cells was measured after $72 \mathrm{~h}$ of culture. Briefly, $500 \mu \mathrm{L}$ samples of conditioned media or nitrite standards $(0-100 \mathrm{nM})$ were mixed with $500 \mu \mathrm{L}$ of Griess reagent ( $1 \%$ sulfanylamide and $0.1 \%$ naphthylethylene-diamine in $5 \%$ phosphoric acid) and absorbance was measured at $530 \mathrm{~nm}$ against a blank prepared with non-conditioned medium. RAW 264.7 cells were also plated on standard culture tissue dishes with lipopolysaccharide (LPS, $0.1 \mu \mathrm{g} / \mathrm{mL}$ ) as positive controls. ${ }^{1}$

\section{Scanning Electron Microscopy (SEM)}

Scaffold surfaces were coated with gold and their morphology was examined using SEM (Phillips 505, Holland), with an accelerating voltage of $20 \mathrm{kV}$. Images were analyzed by Soft Imaging System ADDAII.

\section{$\mathrm{Sr}^{2+}$ Release from PCL-PDIPF Compatibilized Blends}

To determine whether the activity of cells grown on strontium-containing scaffolds might be dependent on $\mathrm{Sr}^{2+}$ released into the culture medium, $\mathrm{Sr}^{2+}$ release was evaluated in a cell-free system. Briefly, the films were cut using a $4 \mathrm{~mm}$ diameter punch, weighed and incubated in $1 \mathrm{ml}$ of DMEM- $10 \%$ FBS at $37{ }^{\circ} \mathrm{C}$ and $5 \% \mathrm{CO}_{2}$. After different incubation times, the medium was collected and $\mathrm{Sr}^{2+}$ was measured using an atomic absorption spectrophotometer (AA-7000, Shimadzu) using a calibration curve made with $\mathrm{Sr}^{2+}$ standards between 0.5 and $2 \mathrm{ppm}$ of $\mathrm{Sr}^{2+}$.

\section{In Vivo Evaluation of Scaffolds With and Without $\mathrm{Sr}^{2+}$}

In the in vivo studies, we evaluated the effect of the PCL/PDIPF compatibilized blend (with or without $1 \% \mathrm{w} / \mathrm{w} \mathrm{Sr}^{2+}$ ) on bone repair using a re-ossification model of critical bone lesion as described previously. ${ }^{34}$
All experiments with animals were done in conformity with the Guidelines on Handling and Training of Laboratory Animals (2011). ${ }^{17}$ Approval for animal studies was obtained from our institutional animal welfare committee (CICUAL Protocol Number 00105-15). Twelve 3-month-old male WKAH/Hok Wistar rats (190-210 g) were maintained in a temperaturecontrolled room at $23{ }^{\circ} \mathrm{C}$ with a fixed $12 \mathrm{~h}$ light: $12 \mathrm{~h}$ darkness cycle and fed standard rat laboratory chow and water ad libitum. For surgical induction of bone lesions, all animals were anesthetized by intraperitoneal/intramuscular injection of $0.12 \mathrm{ml} / 100 \mathrm{~g}$ body weight with $62.5 \mathrm{mg} / \mathrm{mL}$ ketamine hydrochloride and $6.25 \mathrm{mg} / \mathrm{mL}$ xylazine (Laboratorios Richmond, Buenos Aires, Argentina). Circular craniotomy defects of $2.0 \mathrm{~mm}$ diameter were made in both parietal bones of each animal with a cylindrical low-speed carbide bur. All twelve animals were then randomly separated equally into two groups of six: one in which a PCL/ PDIPF scaffold without $\mathrm{Sr}^{2+}$ was implanted in the lesion of the right parietal bone of each animal; and another in which a PCL/PDIPF scaffold with $1 \% \mathrm{Sr}^{2+}$ was implanted in the right craniotomy of each animal. In all cases, the left parietal lesion was allowed to heal without addition of a scaffold (as a counter-lateral internal control for each animal). Four weeks after surgery and prior to sacrifice, non-fasting blood samples were taken from all animals and serum was stored at $-20{ }^{\circ} \mathrm{C}$ until biochemical evaluation (total ALP activity, glucose and creatinine by commercial kits; strontium by atomic absorption). All rats were then sacrificed under anesthesia by rapid cervical dislocation, after which both parietal bones were dissected for histological evaluation of bone re-ossification and for RT-PCR determination of osteogenic markers respectively, as described in the following sections.

\section{Histological Evaluation of Bone}

Dissected parietal bones (4 animals per group) were fixed in $10 \%$ formalin, decalcified in $10 \%$ EDTA, embedded in paraffin and $5 \mu \mathrm{m}$ sections were obtained with an SM 2000R Leica microtome. The sections were stained with hematoxylin-eosin (H-E). Pictures were taken with a Nikon Coolpix 4500 digital camera on an Eclipse E400 Nikon microscope. Images were analyzed using the Image $\mathbf{J}$ program (www.macbiophotonics.c a/imagej) with a Microscope scale plugin. At the site of the surgically induced parietal lesions, bone regeneration was calculated as the ratio between the newly reossified area and the original bone lesion area. In addition, inflammatory response and bridging of the lesion by fibrous tissue were qualitatively evaluated. 


\section{Reverse Transcriptase ( $R T$ )-Polymerase Chain Reaction (PCR) Evaluation of Osteogenic and/or Pro-inflammatory Markers}

For RT-PCR studies, total RNA was isolated from: (a) dissected right and left parietal bones closely trimmed around the lesion area ( 2 animals per group); (b) BMSC grown on scaffolds in osteogenic media for 14 days; or (c) RAW 264.7 cultured on films for 3 days. In the case of BMSC and RAW 264.7 cells, TRIZOL reagent was used as indicated by the manufacturer (Invitrogen, Argentina). For RNA extraction from trimmed parietals, the bone samples were ground in liquid nitrogen after which TRIZOL reagent was added according to manufacturer instructions. Expression of osteogenic markers (ALP, Runx-2) or cytotoxicity markers (TNF- $\alpha$, IL-1, iNOS) was performed by semi-quantitative RT-PCR using MMLVRT (Invitrogen, Argentina). Expression of all markers were normalized to $\beta$-actin (housekeeping gene). Specific primers for all markers were designed from NCBI sequence data using CLC Genomics Workbench software (QIAGEN) (Table 1) and synthesized by Macrogen (Seoul, Republic of Korea). After separation of RT-PCR products by agarose gel electrophoresis with ethidium bromide, their corresponding band intensities were quantified using the gel plugin of MBF_ImageJ program.

\section{Statistical Analysis}

Results are expressed as the mean \pm SEM and, unless indicated otherwise, were obtained from two separate experiments performed in triplicate. Differences between the groups were assessed by one-way ANOVA with Tukey post hoc test. For non-normal distributed data, a non-parametrical Kruskal-Wallis evaluation with Dunn post hoc test was performed using GraphPad In Stat version 3.00 (Graph Pad Software). A $p$ value $<0.05$ was considered significant for all statistical analyses.

\section{RESULTS}

\section{Determination of Scaffold Surface Characteristics by $S E M$}

Figure 1 shows SEM images of the surface of the Blend without $\mathrm{Sr}^{2+}$, with $1 \% \mathrm{Sr}^{2+}$ and with $5 \% \mathrm{Sr}^{2+}$. The Blend without $\mathrm{Sr}^{2+}$ showed a smooth surface with spherulite morphology, as we have previously described. ${ }^{12}$ Blend $+1 \% \mathrm{Sr}^{2+}$ showed a similar surface to Blend, however with a homogeneous dispersion of fine strontium particles on all its surface. On the contrary, Blend $+5 \% \mathrm{Sr}^{2+}$ showed a surface aggregate of strontium particles and a heterogeneous disposition. For both scaffolds, surface aggregates were unaffected by careful sample manipulation, or by 2 -week incubation in culture media (data not shown).

\section{Strontium Release from the Scaffold in a Cell-Free Environment is Negligible}

We used an atomic absorption spectrophotometer to measure free $\mathrm{Sr}^{2+}$ released into culture media after a 14-day incubation with Sr-containing membranes. Figure 2 shows the time-course of $\mathrm{Sr}^{2+}$ release. As can be seen for both composite scaffolds, release was very low: \% accumulative $\mathrm{Sr}^{2+}$ release from Blend $+1 \%$ $\mathrm{Sr}^{2+}$ reached a maximum of $0.005 \%$ after nine days (and a maximum of $0.001 \%$ after 4 days in the case of Blend $+5 \% \mathrm{Sr}^{2+}$ ). This means that most of the strontium is retained within the polymeric matrices.

\section{Strontium Content of the Scaffold Modifies Its In Vitro Osteocompatibility}

Bone marrow stromal cells (BMSC) were grown on the scaffolds in basal medium, and their proliferation was evaluated at different time-points ( $2 \mathrm{~h}$ to 5 days, Fig. 3). BMSC proliferated better on Blend $+1 \%$ $\mathrm{Sr}^{2+}$ than on Blend alone $(p<0.01)$. On the other hand, after 5 days Blend $+5 \% \mathrm{Sr}^{2+}$ significantly decreased BMSC proliferation vs. both Blend alone $(p<0.01)$ and Blend $+1 \% \operatorname{Sr}^{2+}(p<0.01)$.

In further experiments, BMSC were grown for 14 days with an osteogenic medium in order to evaluate the effect of Sr-containing scaffolds on the capacity of these cells to express two bone markers, Alkaline Phosphatase activity (ALP) and type 1 Collagen (Col-1) production. As shown in Fig. 4a, the addition of $1 \% \mathrm{Sr}^{2+}$ didn't modify Col-1 vs. Blend alone. However, incorporation of $5 \% \mathrm{Sr}^{2+}$ significantly decreased Col-1 compared to both Blend alone $(p<0.05)$ and Blend $+1 \% \mathrm{Sr}^{2+}(p<0.05)$. When ALP activity (Fig. 4b) of these cells was evaluated, Blend $+1 \% \mathrm{Sr}^{2+}$ induced an increase in this osteoblastic marker vs. Blend alone $(p<0.05)$, whereas for Blend $+5 \% \mathrm{Sr}^{2+}$ significant decreases were found when compared both with Blend alone $(p<0.01)$ and with Blend $+1 \% \operatorname{Sr}^{2+}(p<0.01)$.

We also evaluated the expression of osteoblastic differentiation markers Runx2 and ALP by RT-PCR. After a 14 day-culture of BMSC in osteogenic media, no differences were observed in the expression of ALP mRNA (Fig. 4c) between cells grown on Blend alone or Blend $+1 \% \mathrm{Sr}^{2+}$. However, this marker was found to be decreased in BMSC cultured on 
TABLE 1. Primer sequences (forward and reverse) for cytotoxic and osteogenic markers.

\begin{tabular}{|c|c|c|c|c|}
\hline Marker & Genbank code & Product size (bp) & & Sequence \\
\hline \multirow[t]{2}{*}{$\beta$-actin } & NM_031144.3 & 345 & fw & ССТTCAACACCCCAGCCAT \\
\hline & & & rv & CATAGCTCTTCTCCAGGGA \\
\hline \multirow[t]{2}{*}{ iNOS } & NM_010927.3 & 499 & fw & ACCAGAGGACCCAGAGACAA \\
\hline & & & rv & CGATGCACAACTGGGTGA \\
\hline \multirow[t]{2}{*}{ IL-1 $\beta$} & NM_008361.3 & 264 & fw & AАGСТСТССАССТСААТG \\
\hline & & & rv & CAGACTCAAACTCСАСTTT \\
\hline \multirow[t]{2}{*}{ TNF- $\alpha$} & NM_013693.3 & 298 & fw & САСGСТСТTСТGTСТАСTG \\
\hline & & & rv & CTTGAAGAGAACCTGGGA \\
\hline \multirow[t]{2}{*}{ ALP } & J03572.1 & 737 & fw & GACAGCAAGCCCAAGAGA \\
\hline & & & rv & CAGTTCAGTGCGGTTCCA \\
\hline \multirow[t]{2}{*}{ RUNX2 } & NM_001278483.1 & 598 & fw & GCCGGGAATGATGAGAACTA \\
\hline & & & rv & TGAGAGAGGAAGGCCAGA \\
\hline
\end{tabular}
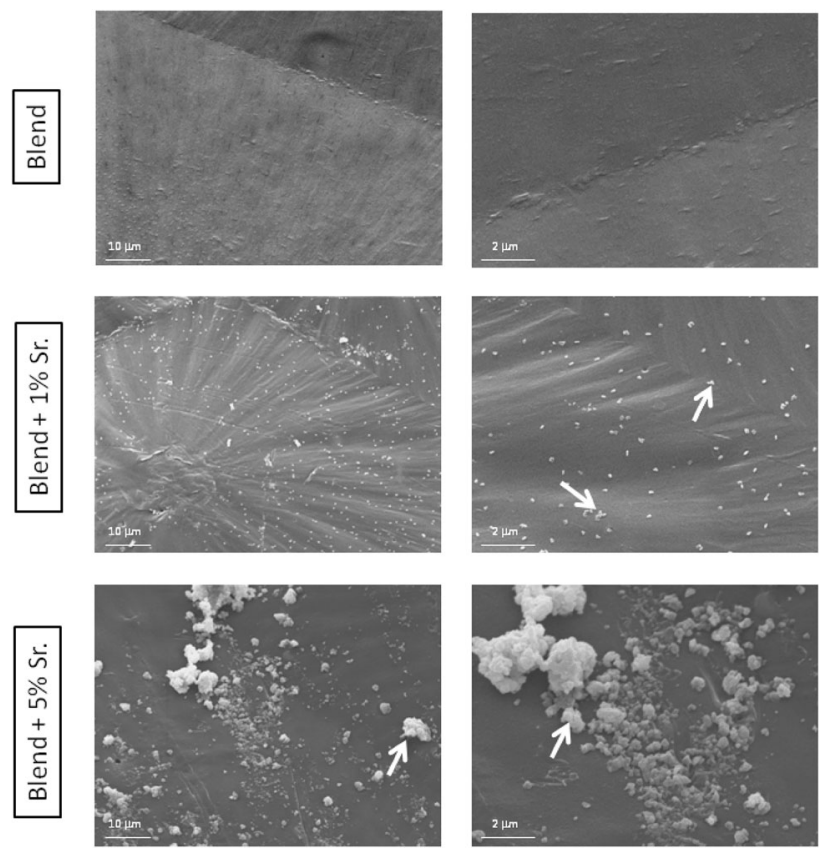

FIGURE 1. SEM images of Blend, Blend $+1 \% \mathrm{Sr}$ and Blend $+5 \% \mathrm{Sr}$ at a magnification of 1000 (left column) and 2500 (right column). White arrows indicate strontium aggregates in polymer.

Blend $+5 \% \mathrm{Sr}^{2+}$. As shown in Fig. 4d, Runx2 expression was increased in cells grown on Blend + $1 \% \mathrm{Sr}^{2+}(p<0.01)$ and decreased in BMSC grown on Blend $+5 \% \mathrm{Sr}^{2+}(p<0.01)$, vs. Blend alone.

\section{Cytotoxicity of the Scaffold is Dependent on Strontium} Dopage of Polymers

To evaluate the possible cytotoxicity of Sr-containing scaffolds, we cultured macrophage-derived RAW 264.7 cells on polymeric films for $72 \mathrm{~h}$, after which NO production (Fig. 5a), as well as the expression of

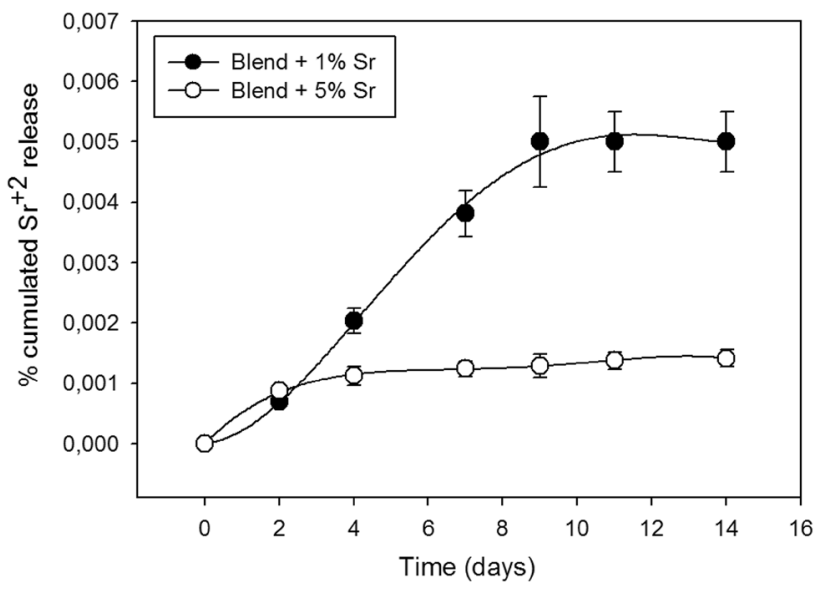

FIGURE 2. Accumulative $\mathrm{Sr}^{2+}$ release (as $\%$ of total $\mathrm{Sr}^{2+}$ in scaffold) into culture media from Blend $+1 \% \mathrm{Sr}^{2+}$ and Blend $+5 \% \mathrm{Sr}^{2+}$, over a period of 14 days.

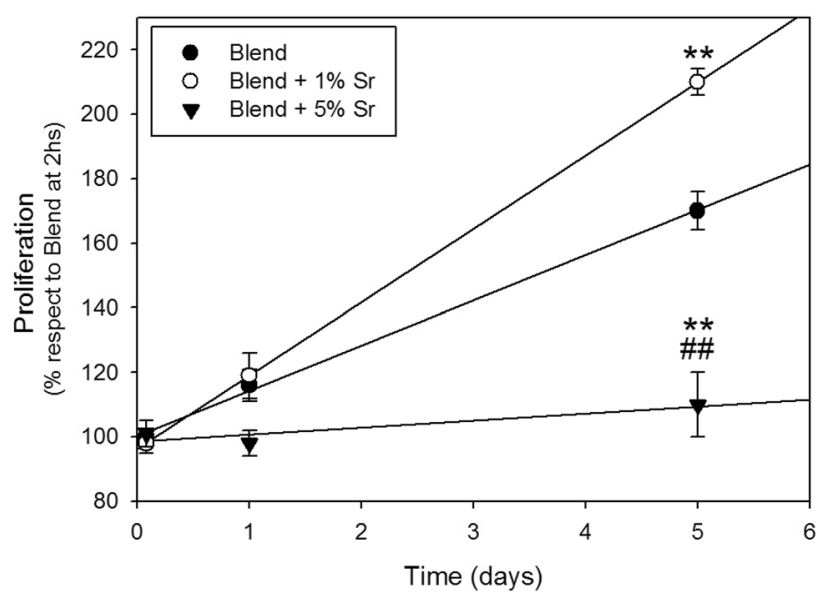

FIGURE 3. Proliferation of bone marrow stromal cells (BMSC) grown on Blend, Blend $+1 \% \mathrm{Sr}^{2+}$ and Blend $+5 \%$ $\mathrm{Sr}^{2+}\left({ }^{\star *} p<0.01\right.$ vs. Blend; ${ }^{\#} p<0.01$ vs. Blend $\left.+1 \% \mathrm{Sr}^{2+}\right)$.

TNF $\alpha$, IL-1 and iNOS mRNA by RT-PCR (Fig. 5b) were determined. As a positive control for cytotoxicity, RAW 264.7 cells were cultured in the presence of 
(a)

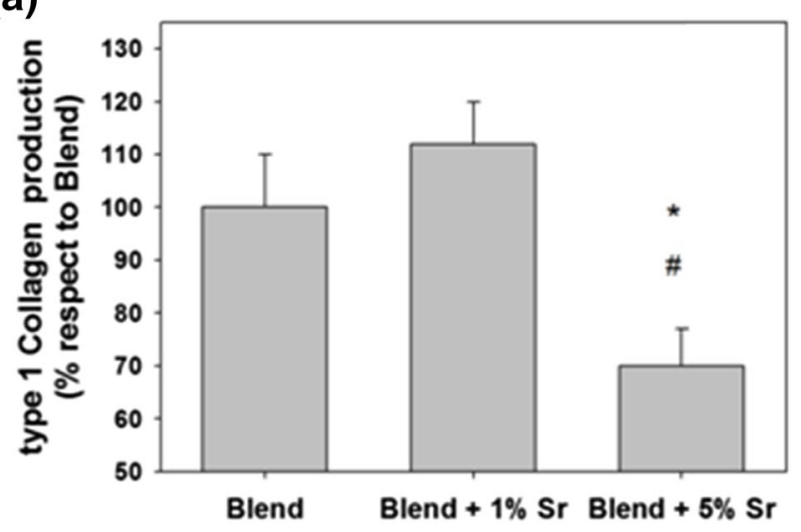

(c)

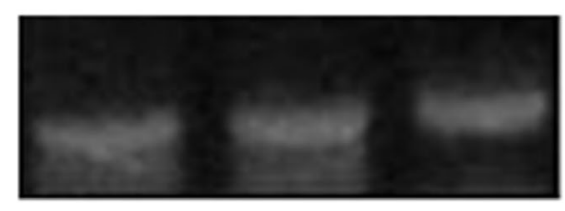

ALP

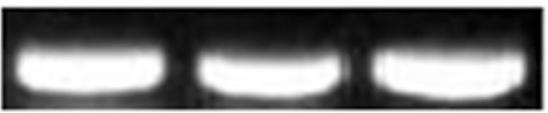

$\beta$-actin (b)

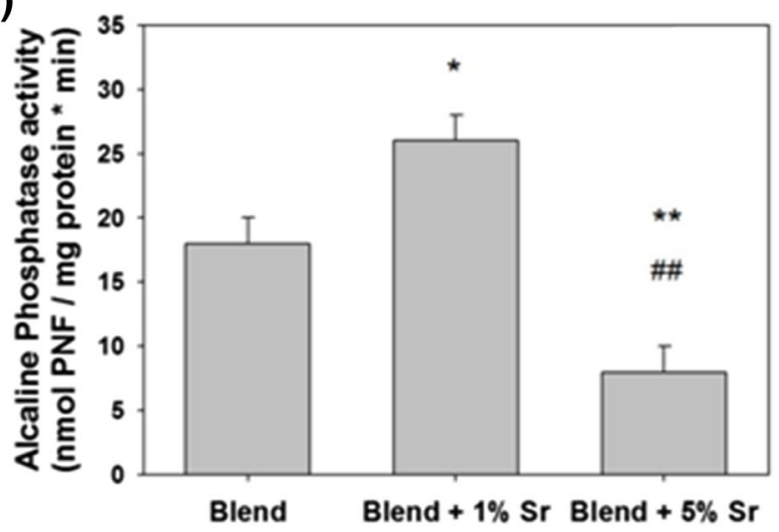

(d)
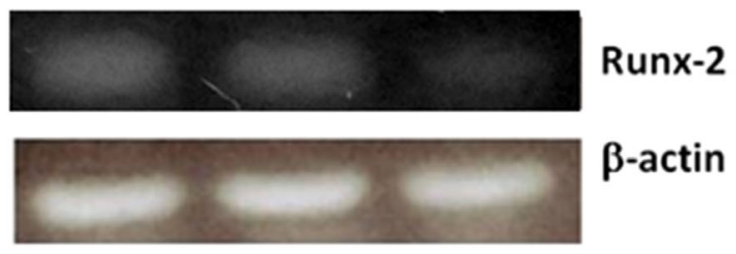

Blend Blend $+1 \% \mathrm{Sr}$ Blend $+5 \% \mathrm{Sr}$

\section{Blend Blend $+1 \% \mathrm{Sr}$ Blend $+5 \% \mathrm{Sr}$}
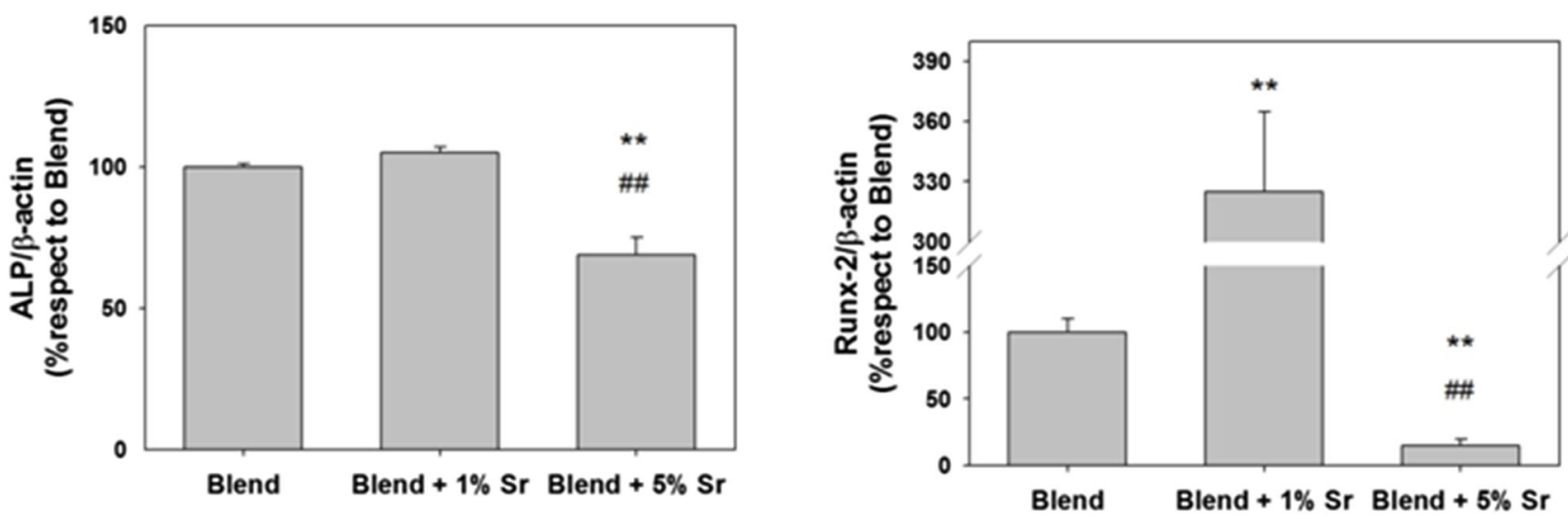

FIGURE 4. Osteoblastic differentiation markers after a 14-day osteogenic culture of $\mathrm{BMSC}$ on $\mathrm{Blend} \mathrm{Blend}^{+1} \% \mathrm{Sr}^{2+}$ and Blend $+5 \% \mathrm{Sr}^{2+}$. Type 1 collagen production (a); alkaline phosphatase activity (b); semi-quantitative analysis of mRNA for alkaline phosphatase (c) and Runx-2 (d) normalized to $\beta$-actin $\left(^{*} p<0.05\right.$ vs. Blend; ${ }^{* \star} p<0.01$ vs. Blend; ${ }^{\#} 0.05$ vs. Blend $+1 \%$ Sr ${ }^{2+}$; $\# p<0.01$ vs. Blend $\left.+1 \% \mathrm{Sr}^{2+}\right)$.

$0.1 \mu \mathrm{g} / \mathrm{mL}$ lipopolysaccharide (LPS). NO released from cells grown on Blend, Blend $+1 \% \mathrm{Sr}^{2+}$ and Blend $+5 \% \mathrm{Sr}^{2+}$ was similar to that of a standard plastic tissue culture dish (data not shown). In addition, they were significantly lower than the positive (LPS) control. Figure 5b shows that there were no significant differences in the levels of iNOS expression of cells grown on the different polymeric scaffolds, or on standard plastic culture dishes. TNF- $\alpha$ and IL1 expression were significantly increased when RAW
264.7 were cultured on Blend $+5 \% \mathrm{Sr}^{2+}$ but were unaltered in BMSC grown on Blend $+1 \% \mathrm{Sr}^{2+}$.

\section{Sr-containing Scaffold Shows an Improved Capacity for In Vivo Bone Repair}

Having determined an improvement in osteocompatibility (vs. Blend alone) and absence of cytotoxicity for Blend $+1 \% \mathrm{Sr}^{2+}$, we proceeded to evaluate the in vivo ability of this scaffold to induce bone repair in a 
(a)

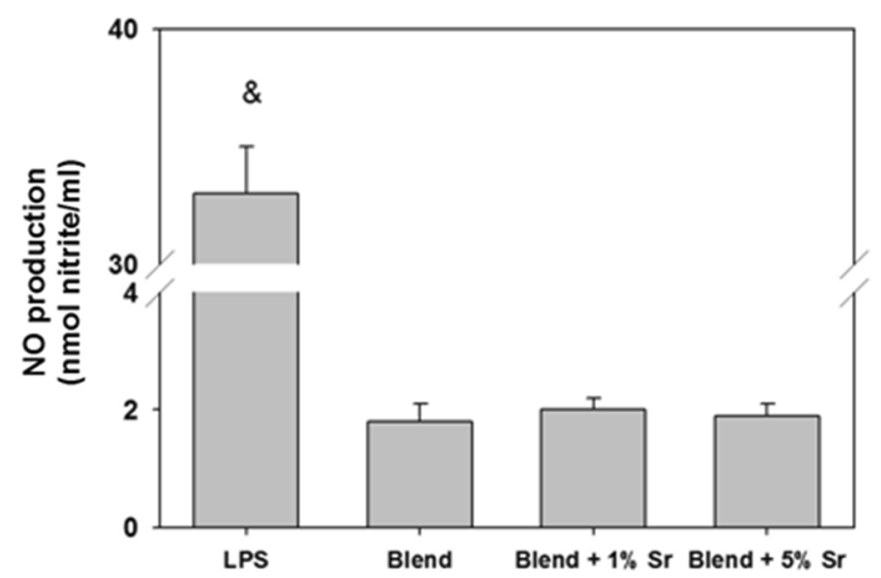

(b)
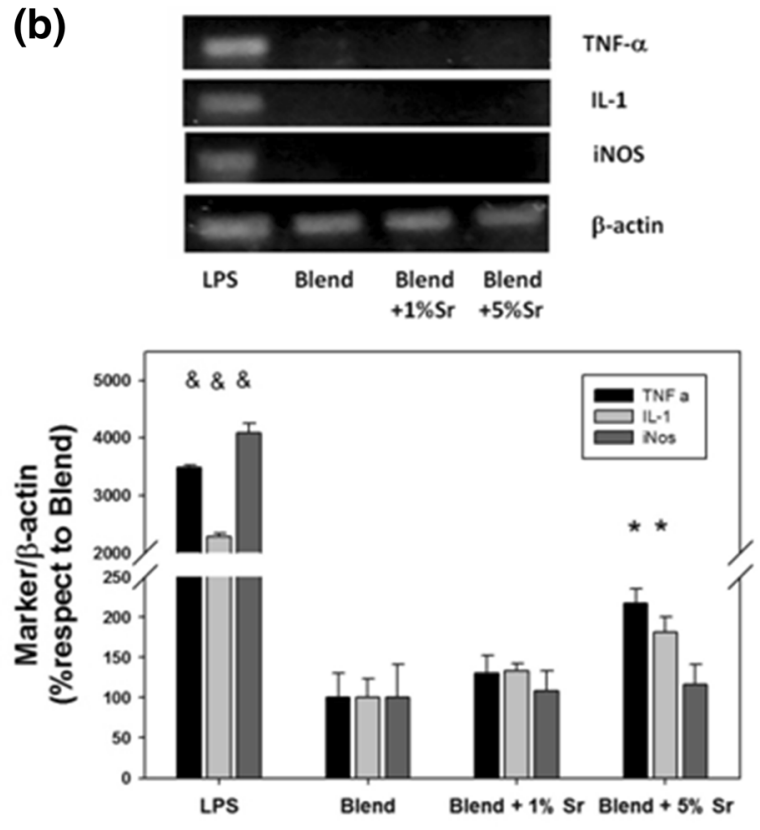

FIGURE 5. Cytotoxicity studies of scaffolds using RAW 264.7 macrophages. NO production (a); semi-quantitative analysis of mRNA for TNF- $\alpha$, IL-1 and iNOS normalized to $\beta$-actin (b) ( ${ }^{\&} p<0.001$ vs. non-LPS; ${ }^{\#} p<0.05$ vs. Blend and Blend $+1 \%$ Sr $\left.{ }^{2+}\right)$.

surgically-induced critical parietal lesion. In each animal, lesions were performed bilaterally (in one craniotomy a scaffold was implanted immediately after surgery, while the other was allowed to heal unaided as an internal control). At the end of the experiments, no significant differences were observed for both groups of animals in serum ALP activity, and in glucose, creatinine or strontium levels (data not shown). As can be seen in the histomorphometric evaluation shown in Figs. 6a-6d, the 4-week period allowed for bone repair was insufficient for a complete bridging of the defect with bone tissue. In lesions without an implant, relative bone regeneration was $35 \pm 1 \%$ of the original lesion area, with bridging completed by abundant fibrous tissue. Implant of scaffolds with Blend alone, although stabilizing the lesion and visibly un-degraded over a 4week repair period (important properties for lesions of load-bearing bones), however approximately halved relative bone re-ossification and reduced the thickness of the intervening fibrous tissue. Interestingly, implant of a scaffold with Blend $+1 \% \mathrm{Sr}^{2+}$ (also virtually undegraded after 4 weeks) increased relative bone repair vs. Blend alone by $52 \%(p<0.01)$, while also improving fibrous bridging. In all parietal lesions with implants, absence of a scaffold-induced inflammatory reaction was verified.

A similar pattern of results was obtained when we evaluated the expression of Runx2 (a positive regulator of osteogenesis) by RT-PCR in the parietal lesions (Fig. 6e). Runx 2 mRNA was approximately halved by the implant of a scaffold with Blend alone (vs. un- implanted control) but increased significantly in the presence of Blend $+1 \% \mathrm{Sr}^{2+}$ (vs. Blend alone).

\section{DISCUSSION}

Various polymers are being intensely developed and studied to determine their potential use for bone tissue engineering. We have previously developed and characterized a compatibilized Blend of PCL and PDIPF. $^{12,15}$ We performed no tests to evaluate porosity; however, these membranes should not be considered porous because they have been obtained by the casting technique. Strontium is a divalent cation that can partially substitute $\mathrm{Ca}^{2+}$ in bone hydroxyapatite crystal lattice. Although not calcium-mimetic, $\mathrm{Sr}$ cations can interact with cellular calcium-sensing receptors to differentially activate both anabolic and anti-resorptive effects on bone metabolism. ${ }^{23}$ Thus, in the present work we attempted to improve the osteoinductive properties of our scaffold, by incorporating strontium $(1 \%$ or $5 \%)$ into the PCL-PDIPF Blend.

Strontium ranelate (SR) has been used for more than 10 years in many countries (though not the USA) as a first-line treatment for post-menopausal osteoporosis. $^{33} \mathrm{We}$ have previously demonstrated that $0.1 \mathrm{mM}$ of strontium (as SR) increases the proliferation and ALP activity of MC3T3-E1 pre-osteoblastic cells. ${ }^{15}$ In line with these results, Almeida and collaborators found that SR promotes the proliferation, type

\section{BMES



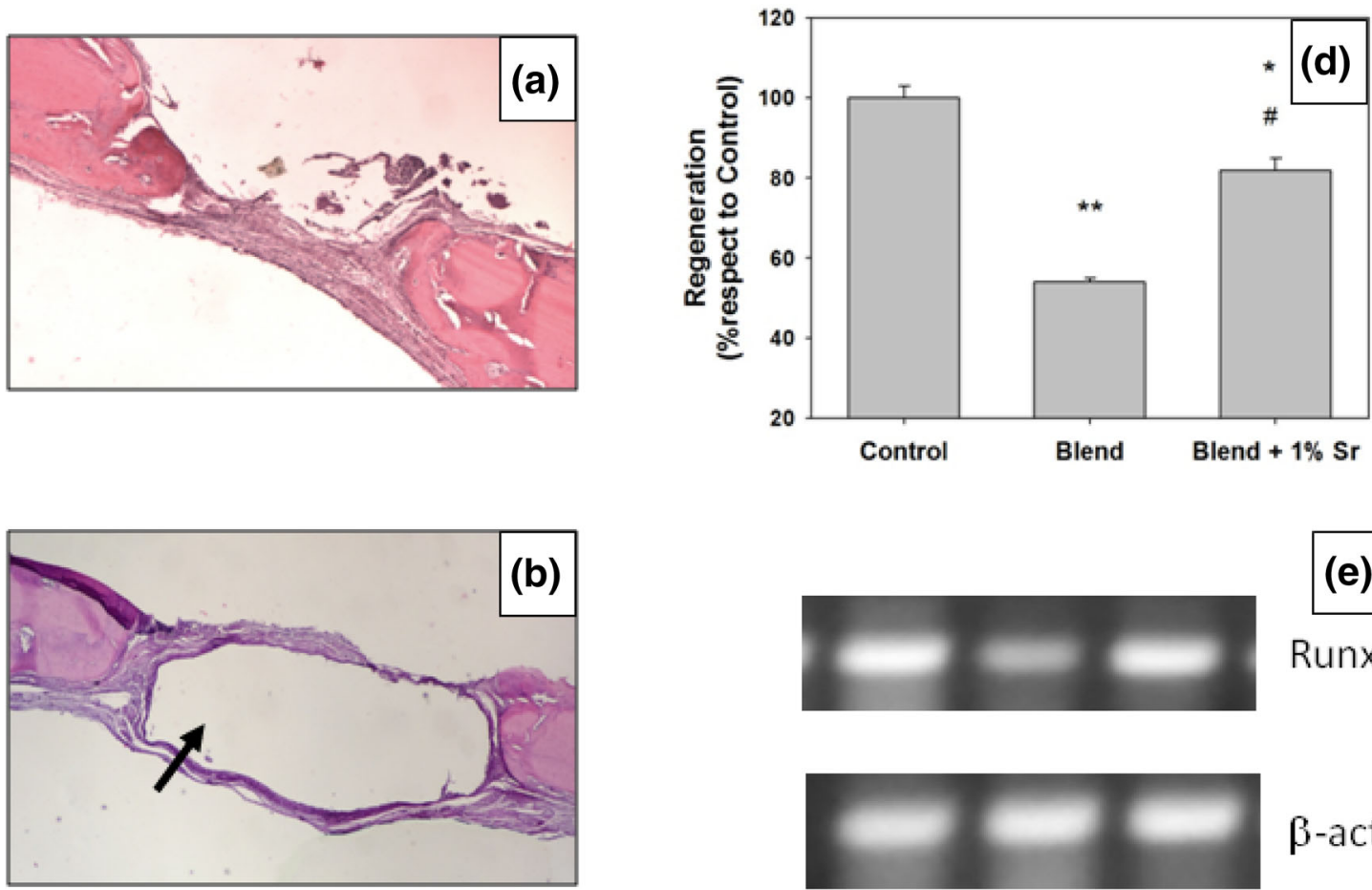

(e)

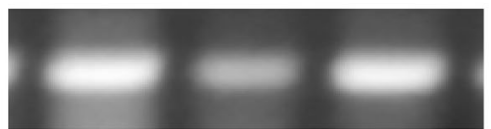

Runx-2
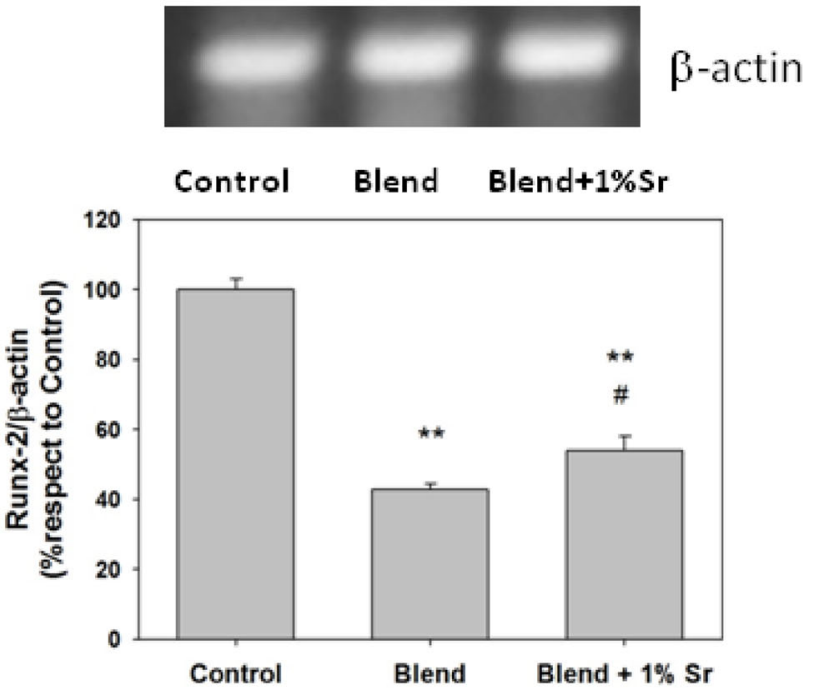

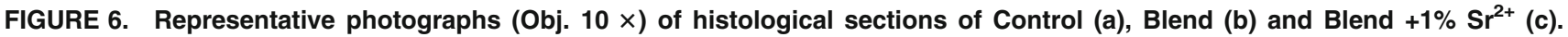
Quantitation of \% bone regeneration, as defined in "Materials and Methods" (d). Semi-quantitative analysis of mRNA for Runx-2 normalized to $\beta$-actin (e). ( ${ }^{*} p<0.05$ vs. un-implanted control; ${ }^{* \star} p<0.01$ vs. un-implanted control; ${ }^{\#} 0.05$ vs. Blend). Black arrows indicate remains of polymeric implants.

1 collagen production and mineral nodule deposition of MC3T3-E1 cells. ${ }^{2}$ We have observed that SR can also promote the osteogenic differentiation of vascular smooth muscle cells in vitro, identifying a potentially pro-atherogenic cellular mechanism induced by SR. ${ }^{26}$ Recently, serious clinical adverse effects for SR use were identified, and in 2014 the European Medicine Agency recommended discontinuation of SR treatments in patients with a history of hypertension or cardiovascular disorder. ${ }^{10}$ In 2016, Bolland and Grey reviewed the adverse cardiovascular effects of SR treatment. ${ }^{3}$ As of August 2017, Servier decided to discontinue distribution of SR worldwide. Thus, in our present work it was important to show that strontiumcontaining scaffolds did not release significant concentrations of this cation (i.e., that the release of $\mathrm{Sr}^{2+}$ was negligible compared with the concentrations needed to induce any of its cardiovascular systemic effects, while permitting local effects on bone cells at the bonescaffold interface).

After incubating the scaffolds in a cell-free system, the maximum concentration of $\mathrm{Sr}^{2+}$ released into the culture medium was $0.7 \pm 0.1 \mathrm{ppm}$ (for Blend $+5 \%$ $\mathrm{Sr}^{2+}$ after 2 days of incubation). Although these values are very low $\left(8 \%\right.$ of the plasma $\mathrm{Sr}^{2+}$ concentration that is clinically relevant, and of culture media levels 
that we have previously shown to be optimally osteogenic in vitro), ${ }^{15}$ Blend $+1 \% \mathrm{Sr}^{2+}$ and Blend $+5 \%$ $\mathrm{Sr}^{2+}$ respectively induced an increase and a decrease in the osteogenic markers of BMSC growing on them. A possible explanation for these effects could be a significantly greater concentration of $\mathrm{Sr}^{2+}$ in the cellular micro-environment (i.e., at the cell-scaffold interface) than in the culture medium. This concept is in agreement with the observation of strontium aggregates on the surface of these scaffolds (Fig. 1). Importantly, serum levels of $\mathrm{Sr}^{2+}$ did not differ significantly between animals with implants of Sr-containing and non-Sr-containing scaffolds for 4 weeks in this study.

Recently, Meka et al. developed a scaffold with PCL and $\mathrm{Sr}^{2+}$ using an electrospinning technique. They measured $\mathrm{Sr}^{2+}$ release from the scaffold and found that it peaked at 4 days, decreasing thereafter. On the fourth day a maximum concentration of $60 \mathrm{ppm}$ of $\mathrm{Sr}^{2+}$ was attained in the culture medium, which was able to promote the osteogenic differentiation of human mesenchymal stem cells in vitro. ${ }^{24}$ Nair and collaborators designed another strontium ranelate delivery system using a PCL-laponine composite. These authors reported up to $40 \%$ accumulative release of strontium from their material, ${ }^{29}$ reaching $\mathrm{Sr}^{2+}$ levels in the culture medium that were capable of increasing the ALP activity of human osteosarcoma cells in vitro. Clearly, $\mathrm{Sr}^{2+}$ release into the extracellular medium (an important consideration for its clinical applicability in BTE) depends on the structural composition of the scaffold.

Another very important requirement for biomaterials is that they must be non-toxic. To evaluate the possible toxicity of our Sr-containing scaffolds, we used RAW 264.7 cells. This cell line is derived from mouse (Mus musculus) macrophages. These cells are adherent and possess monocytic morphology. ${ }^{32}$ They express different markers of macrophage activity, such as interleukin synthesis, nitric oxide (NO) production and expression of nitric oxide synthases (NOS) when exposed to toxic substances. ${ }^{9}$ Due to these characteristics, they constitute an excellent model for studies of cytotoxicity of different substances in biological systems.

In the present study, we were only able to find an increase in the expression of pro-inflammatory cytokines, after exposure of the macrophages to a scaffold with Blend $+5 \% \mathrm{Sr}^{2+}$ for 3 days. This increased expression of TNF- $\alpha$ and IL- 1 suggests that for this particular scaffold, the concentration of strontium in the cellular microenvironment is sufficiently high to induce a toxic effect. Our release studies show that these toxic effects cannot be a consequence of the low levels of strontium released into the culture medium. Most probably, they are due to a direct interaction between cells and strontium aggregates on the polymeric surface. This cytotoxic effect could also be the cause of the decreased in vitro osteogenic induction observed for BMSC grown on Blend $+5 \%$ $\mathrm{Sr}^{2+}$ (vs. Blend alone and Blend $+1 \% \mathrm{Sr}^{2+}$ ).

In further in vitro experiments, Blend $+1 \% \mathrm{Sr}^{2+}$ showed an improvement in its osteocompatibility vs. Blend alone (significant increases in the proliferation, in the expression of Runx2, and in the ALP activity of cultured BMSC). In previous in vitro studies, we have shown a direct effect of different $\mathrm{Sr}^{2+}$ concentrations on the ALP activity of UMR 106 osteoblastic cell lysates. Specifically, the Km for ALP decreased with the addition of $0.1 \mathrm{mM}$ of $\mathrm{Sr}^{2+}$, thus increasing the apparent enzymatic activity of this enzyme. ${ }^{14}$ The results obtained in the present study for bone-specific Alkaline phosphatase of BMSC grown on Blend + $1 \% \mathrm{Sr}^{2+}$ (i.e., an increase in ALP enzymatic activity but not in its gene expression) can be explained by a Srinduced decrease in the $\mathrm{Km}$ of this enzyme rather than by regulation at a genetic level.

In all, our in vitro experiments show that addition of $1 \% \mathrm{Sr}^{2+}$ to the PCL-PDIPF Blend increases its osteocompatibility without any cytotoxic effects or significant release of the cation; whereas addition of $5 \% \mathrm{Sr}^{2+}$ induces both anti-osteogenic and proinflammatory effects. On the basis of this evidence, we selected scaffolds with Blend $+1 \% \mathrm{Sr}^{2+}$ for in vivo experiments to evaluate the efficacy of this biomaterial as an implant to aid in the repair of surgically-induced bone lesions.

In a recent article, Neves et al. reviewed 27 published reports evaluating in vivo bone regeneration aided by different Sr-containing biomaterials. ${ }^{30}$ These authors conclude that although Sr-enriched scaffolds are apparently safe and effective for BTE, care must be taken to prevent adverse systemic side-effects of $\mathrm{Sr}^{2+}$. Their conclusions are in line with the in vivo results of our present study, in which: (a) we found no difference in serum $\mathrm{Sr}^{2+}$ levels between animals with $\mathrm{Sr}$-containing and non-Sr-containing implants; (b) implants did not induce a local inflammatory response; and (c) vs. the PCL-PDIPF scaffold alone, enrichment of the biomaterial with $1 \% \mathrm{Sr}^{2+}$ significantly increased expression of the osteoblastic transcription factor Runx 2 as well as relative bone tissue regeneration and fibrous bridging. However, Blend $+1 \% \mathrm{Sr}^{2+}$ induced lower relative bone regeneration than absence of polymer (counter-lateral control). In future experiments, we will evaluate the efficacy of porous scaffolds with $1 \% \mathrm{Sr}^{2+}$ to repair bone lesions, taking care that porosity does not significantly compromise mechanical stability.

Our present results have several limitations. To begin with, we have not used a model of lesion in a 
weight-supporting bone, in which the use of a scaffold with adequate mechanical properties is important for lesion stabilization. Secondly, the biomaterial used for implant in our studies was not porous, and this way may have limited the speed of bone regeneration. In addition, we did not study the mechanism by which strontium is retained by the polymer rather than being released into culture media (we speculate that strontium-containing aggregates may be coated by a thin film of polymer that could prevent fast delivery of this cation, while allowing local interaction with cells). Finally, the number of animals used in our present study was relatively small. Larger-scale experiments are currently under way in our laboratory to address these issues.

In conclusion, we have enriched a compatibilized blend of PCL-PDIPF with $1 \% \mathrm{Sr}^{2+}$ and evaluated the applicability of this biomaterial for bone tissue engineering (BTE), both in vitro and in vivo. In vitro, the Blend $+1 \% \mathrm{Sr}^{2+}$ (a) released very low quantities of the cation; (b) was not cytotoxic for cultured macrophages; and (c) showed improved osteocompatibility (vs. Blend alone) when used as a substratum for primary cultures of bone marrow stromal cells. In vivo (in a surgically-induced critical bone lesion), implants with Blend $+1 \% \mathrm{Sr}^{2+}$ significantly increased bone tissue regeneration and improved fibrous bridging (vs. Blend alone), while neither inducing a local inflammatory response nor an increase in serum levels of $\mathrm{Sr}^{2+}$. Altogether, these results indicate that our compatibilized blend of PCL-PDIPF enriched with $1 \% \mathrm{Sr}^{2+}$ could be useful for BTE, although additional studies are necessary to determine its efficacy and safety in a clinical setting.

\section{ACKNOWLEDGMENTS}

ABL is a Fellow of Consejo Nacional de Investigaciones Científicas y Técnicas (CONICET), Argentina. ADM is a part-time full Professor of Clinical Chemistry at National University of La Plata (UNLP), Argentina. JMF is a Member of the Carrera del Investigador of Consejo Nacional de Investigaciones Científicas y Técnicas (CONICET), Argentina. This study was supported by ANPCyT grants (PICT 20120053 to ADM, PICT 2015-1361 to ADM and PICT 2015-1030 to JMF).

\section{CONFLICT OF INTEREST}

All authors declare that they have no conflicts of interest.

\section{REFERENCES}

${ }^{1}$ Alfano, A. L., and J. M. Fernandez. Induction of topographical changes in PCL scaffolds for bone tissue engineering: biocompatibility and cytotoxicity evaluations. $J$. Biomater. Tissue Eng. 5:142-149, 2015.

${ }^{2}$ Almeida, M. M., E. P. Nani, L. N. Teixeira, D. C. Peruzzo, J. C. Joly, M. H. Napimoga, and E. F. Martinez. Strontium ranelate increases osteoblast activity. Tissue Cell 48:183-188, 2016.

${ }^{3}$ Bolland, M., and A. Grey. Ten years too long: strontium ranelate, cardiac events, and the European Medicines Agency. BMJ 354:i5109-i5117, 2016.

${ }^{4}$ Braddock, M., M. P. Houston, C. Campbell, and P. P. Ashcroft. Born again bone: tissue engineering for bone repair. Physiology 16:208-213, 2001.

${ }^{5}$ Bradford, M. M. A rapid and sensitive method for the quantitation of microgram quantities of protein utilizing the principle of protein-dye binding. Anal. Biochem. 72:248-254, 1976.

${ }^{6}$ Cortizo, M. S. Polymerization of diisopropyl fumarate under microwave irradiation. J. Appl. Polym. Sci. 103:3785-3791, 2007.

${ }^{7}$ Cortizo, A. M., and S. B. Etcheverry. Vanadium derivatives act as growth factor-mimetic compounds upon differentiation and proliferation of osteoblast-like UMR106 cells. Mol. Cell. Biochem. 145:97-102, 1995.

${ }^{8}$ Cortizo, M. S., M. S. Molinuevo, and A. M. Cortizo. Biocompatibility and biodegradation of polyester and polyfumarate based-scaffolds for bone tissue engineering. J. Tissue Eng. Regen. Med. 2:33-42, 2008.

${ }^{9}$ Denlinger, L. C., P. L. Fisette, K. A. Garis, G. Kwon, A. Vazquez-Torres, A. D. Simon, B. Nguyen, R. A. Proctor, P. J. Bertics, and J. A. Corbett. Regulation of inducible nitric oxide synthase expression by macrophage purinoreceptors and calcium. J. Biol. Chem. 271:337-342, 1996.

${ }^{10}$ European Medicines Agency. PRAC recommends suspending use of Protelos/Osseor (strontium ranelate). http:// www.ema.europa.eu/docs/en_GB/document_library/Refer rals_document/Protelos_and_Osseor/Recommendation_pr ovided_by_Pharmacovigilance_Risk_Assessment_Commit tee/WC $500159374 . p d f[10 / 06 / 2014]$.

${ }^{11}$ Fernandez, J. M., M. S. Cortizo, and A. M. Cortizo. Fumarate/ceramic composite based scaffolds for tissue engineering: evaluation of hydrophylicity, degradability, toxicity and biocompatibility. J. Biomater. Tissue Eng. 4:227-234, 2014.

${ }^{12}$ Fernandez, J. M., M. S. Molinuevo, M. S. Cortizo, and A. M. Cortizo. Development of an osteoconductive PCLPDIPF-hydroxyapatite composite scaffold for bone tissue engineering. J. Tissue Eng. Regen. Med. 5:e126-e135, 2011.

${ }^{13}$ Fernandez, J. M., M. S. Molinuevo, A. M. Cortizo, A. D. McCarthy, and M. S. Cortizo. Characterization of poly(epsilon-caprolactone)/polyfumarate blends as scaffolds for bone tissue engineering. J. Biomater. Sci. Polym. Ed. 21:1297-1312, 2010.

${ }^{14}$ Fernandez, J. M., M. S. Molinuevo, A. D. McCarthy, and A. M. Cortizo. Strontium ranelate stimulates the activity of bone-specific alkaline phosphatase: interaction with $\mathrm{Zn}^{2+}$ and $\mathrm{Mg}^{2+}$. Biometals 27:601-607, 2014.

${ }^{15}$ Fernandez, J. M., M. S. Molinuevo, C. Sedlinsky, L. Schurman, A. M. Cortizo, and A. D. McCarthy. Strontium ranelate prevents the deleterious action of advanced glycation endproducts on osteoblastic cells via calcium channel activation. Eur. J. Pharmacol. 706:41-47, 2013. 
${ }^{16}$ Grillo, C. A., M. A. Reigosa, and M. A. Fernandez Lorenzo de Mele. Does over-exposure to copper ions released from metallic copper induce cytotoxic and genotoxic effects on mammalian cells? Contraception 81:343-349, 2010.

${ }^{17}$ Guidelines on Handling and Training of Laboratory Animals. Published by the Universities Federation for Animals Welfare Guidelines on Handling and Training of Laboratory Animals. In: The Biological Council of Animal Research, Welfare Panel. Guide for the Care and Use of Laboratory Animals: Eighth Edition, edited by Purl UFA. Washington, DC: The National Academies Press, 2011.

${ }^{18}$ Hedberg, E. L., C. K. Shih, J. J. Lemoine, M. D. Timmer, M. A. K. Liebschner, J. A. Jansen, and A. G. Mikos. In vitro degradation of porous poly(propylene fumarate)/ poly(DL-lactic-co-glycolic acid) composite scaffolds. Biomaterials 26:3215-3225, 2005.

${ }^{19}$ Karageorgiou, V., and D. Kaplan. Porosity of 3D biomaterial scaffolds and osteogenesis. Biomaterials 26:54745491, 2005.

${ }^{20}$ Langer, R., and J. Vacanti. Tissue engineering. Science 260:920-926, 1993.

${ }^{21}$ Liu, X., and P. X. Ma. Polymeric scaffolds for bone tissue engineering. Ann. Biomed. Eng. 32:477-486, 2004.

${ }^{22}$ Ma, P. X., R. Zhang, G. Xiao, and R. Franceschi. Engineering new bone tissue in vitro on highly porous $\operatorname{poly}(\alpha-$ hydroxyl acids)/hydroxyapatite composite scaffolds. $J$. Biomed. Mater. Res. 54:284-293, 2001.

${ }^{23}$ Marie, P. J. Strontium ranelate in osteoporosis and beyond: identifying molecular targets in bone cell biology. Mol. Interv. 10:305-312, 2010.

${ }^{24}$ Meka, S. R. K., S. Jain, and K. Chatterjee. Strontium eluting nanofibers augment stem cell osteogenesis for bone tissue regeneration. Colloids Surf. B 146:649-656, 2016.

${ }^{25}$ Molinuevo, M. S., S. B. Etcheverry, and A. M. Cortizo. Macrophage activation by a vanadyl-aspirin complex is dependent on L-type calcium channel and the generation of nitric oxide. Toxicology 210:205-212, 2005.

${ }^{26}$ Molinuevo, M. S., J. M. Fernandez, A. M. Cortizo, A. D. McCarthy, L. Schurman, and C. Sedlinsky. Advanced glycation end products and strontium ranelate promote osteogenic differentiation of vascular smooth muscle cells in vitro: preventive role of vitamin D. Mol. Cell. Endocrinol. 450:94-104, 2017.

${ }^{27}$ Molinuevo, M. S., L. Schurman, A. D. McCarthy, A. M. Cortizo, M. J. Tolosa, M. V. Gangoiti, V. Arnol, and C. Sedlinsky. Effect of metformin on bone marrow progenitor cell differentiation: in vivo and in vitro studies. J. Bone Miner. Res. 25:211-221, 2010.

${ }^{28}$ Morosano, M., A. Masoni, and A. Sánchez. Incidence of hip fractures in the city of Rosario, Argentina. Osteoporos Int. 16:1339-1344, 2005.

${ }^{29}$ Nair, B. P., M. Sindhu, and P. D. Nair. Polycaprolactonelaponite composite scaffold releasing strontium ranelate for bone tissue engineering applications. Colloids Surf. B 143:423-430, 2016.

${ }^{30}$ Neves, N., D. Linhares, G. Costa, C. C. Ribeiro, and M. A. Barbosa. In vivo and clinical application of strontium-enriched biomaterials for bone regeneration: a systematic review. Bone Joint Res. 6:366-375, 2017.

${ }^{31}$ Porter, J. R., T. T. Ruckh, and K. C. Popat. Bone tissue engineering: a review in bone biomimetics and drug delivery strategies. Biotechnol. Progr. 25:1539-1560, 2009.

${ }^{32}$ Raschke, W. C., S. Baird, P. Ralph, and I. Nakoinz. Functional macrophage cell lines transformed by Abelson leukemia virus. Cell 15:261-267, 1978.

${ }^{33}$ Reginster, J. Y., E. Seeman, M. C. De Vernejoul, S. Adami, J. Compston, C. Phenekos, J. P. Devogelaer, M. D. Curiel, A. Sawicki, S. Goemaere, O. H. Sorensen, D. Felsenberg, and P. J. Meunier. Strontium ranelate reduces the risk of nonvertebral fractures in postmenopausal women with osteoporosis: treatment of Peripheral Osteoporosis (TROPOS) Study. J. Clin. Endocrinol. Metab. 90:2816-2822, 2005.

${ }^{34}$ Santana, R. B., X. Lei, B. C. Hermik, A. Salomon, D. T. Graves, and P. C. Trackman. A role for advanced glycation end products in diminished bone healing in type 1 diabetes. Diabetes 52:1502-1510, 2003. 